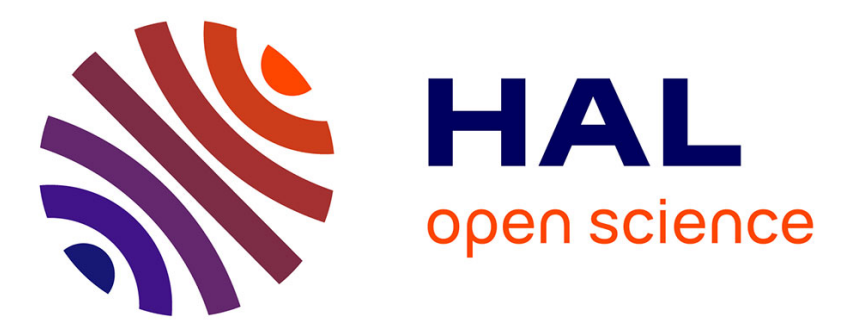

\title{
Capacité statique des transducteurs interdigités à couches minces répartition des charges et des potentiels
}

\author{
J. Duclos, M. Leduc
}

\section{To cite this version:}

J. Duclos, M. Leduc. Capacité statique des transducteurs interdigités à couches minces répartition des charges et des potentiels. Revue de Physique Appliquée, 1982, 17 (2), pp.75-82. 10.1051/rphysap:0198200170207500 . jpa-00244974

\section{HAL Id: jpa-00244974 https://hal.science/jpa-00244974}

Submitted on 1 Jan 1982

HAL is a multi-disciplinary open access archive for the deposit and dissemination of scientific research documents, whether they are published or not. The documents may come from teaching and research institutions in France or abroad, or from public or private research centers.
L'archive ouverte pluridisciplinaire HAL, est destinée au dépôt et à la diffusion de documents scientifiques de niveau recherche, publiés ou non, émanant des établissements d'enseignement et de recherche français ou étrangers, des laboratoires publics ou privés. 
Classification

Physics Abstracts

$41.10 \mathrm{D}-43.88$

\title{
Capacité statique des transducteurs interdigités à couches minces répartition des charges et des potentiels
}

\author{
J. Duclos et M. Leduc (*) \\ Laboratoire d'Electronique et d'Automatique, U.E.R. Sciences et Techniques, B.P. 4006, \\ 76077 Le Havre Cedex, France
}

(*) Laboratoire de Traitement de l'Information

(Reçu le 15 juillet 1981, révisé le 14 octobre 1981, accepté le 20 octobre 1981)

\begin{abstract}
Résumé. - Nous donnons, dans le cas d'un transducteur interdigité à couche mince, une méthode de calcul des potentiels et charges créés en appliquant une tension constante à ses doigts. La méthode repose sur le développement en série de Fourier de différentes fonctions et la traduction des conditions de continuité par un système linéaire dont les inconnues sont les coefficients de Fourier et la capacité de la structure. La méthode présente le double avantage de la précision (incertitude sur la capacité inférieure à $1 \%$ ) et de la généralité (applicable aux systèmes sans couche ou multicouches).

Abstract. - We present a method for calculating potentials and static charges resulting from a voltage applied between fingers and applicable to thin-layered interdigital transducers. The method is based upon a Fourier development of several functions and upon the writing of boundary conditions in a linear system whose unknowns are Fourier coefficients and structure capacitance. The advantages of this method are : precision (capacitances are obtained with accuracy better than $1 \%$ ) and versatility (valid for multi or non layered structures).
\end{abstract}

1. Introduction. - Les dispositifs à ondes de surface acoustiques, de type Rayleigh, sont couramment utilisés dans les lignes à retard, filtres, oscillateurs et dispositifs de traitement du signal tels que corrélateurs [1].

La base de ces matériels est le transducteur, réseau d'électrodes alternées, sur un substrat piézoélectrique, convertissant l'énergie électrique en énergie mécanique de propagation. Pour engendrer des ondes de surface sur un matériatu amorphe, on le recouvre d'une couche mince piézoélectrique et les électrodes sont disposées soit sur la couche, soit à l'interface substratcouche [2,3].

L'excitation des ondes par ce dispositif peut être précisée par l'évaluation des potentiels au niveau des doigts métalliques. On peut, notamment, prévoir l'excitation d'harmoniques ainsi que Engan [4] l'a déjà montré dans un cas particulier.

Pour l'adaptation électrique des transducteurs il est nécessaire de connaître leur capacité. Les travaux de nos prédécesseurs fournissent des résultats volon- tairement approchés par souci de simplicité $[5,6]$ ou par besoin d'expressions algébriques de la capacité [7].

Toutefois Engan [4] a calculé exactement des capacités, mais uniquement dans le cas d'un transducteur sans couche. Quant aux résultats précis de Farnell [8], ils ont été obtenus par une méthode sophistiquée nécessitant d'importants moyens de calcul.

La résolution proposée suppose quelques simplifications usuelles :

- couche monocristalline dont la piézoélectricité ne perturbe pas sensiblement les champs et charges créés par la tension appliquée,

- périodicité des électrodes, supposées d'épaisseur et de résistivité nulles et de longueur infinie (Fig. 1).

Le travail s'effectue en trois étapes :

- on commence par décomposer le potentiel électrique en série de Fourier,

- les équations de continuité sont écrites, aux deux interfaces, en introduisant une fonction charge, définie 
dans le plan contenant les électrodes. Quatre cas sont alors envisagés selon les possibilités de métallisation,

- en précisant les conditions spécifiques imposées, le problème est réduit à une seule dimension (cas monointerface) et se traduit par un système linéaire dont l'ordre peut être choisi selon la précision souhaitée. Sa résolution fournıt finalement la capacité du transducteur et les fonctions décrivant le dispositif.

2. Potentiels. - 2.1 Le DisPositif. - Le dispositif étudié est constitué de trois milieux superposés :

- un substrat isotrope, $s$, d'épaisseur infinie,

- une couche monocristalline, $c$, d'épaisseur constante $H$. Ici nous utilisons un milieu hexagonal d'axe c perpendiculaire à la surface,

- le vide, v, ou l'air.

Le repère est précisé figure 1.

Des électrodes, supposées infiniment longues selon $o x_{2}$, infiniment minces selon $o x_{3}$ et de largeur $d$, sont réparties à intervalle régulier, alternativement aux potentiels $\pm \frac{V}{2} \exp (j \omega t)$. On note $L$ la période des électrodes de même potentiel et $a$ le coefficient de métallisation

$$
0<a=\frac{2 d}{L}<1
$$

On dispose les électrodes dans le plan $x_{3}=0$ ou dans le plan $x_{3}=-H$; de plus on peut envisager la métallisation de l'interface non utilisée. On sait, en effet que cette opération peut modifier de façon avantageuse la génération d'ondes de surface pour de faibles épaisseurs de couche [2].

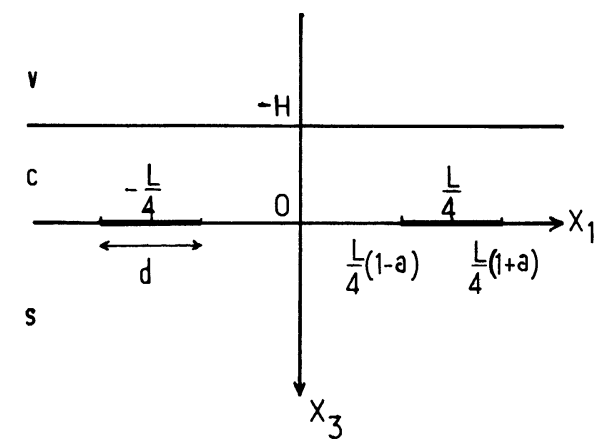

Fig. 1. - Géométrie du transducteur interdigité.

[Interdigital transducer configuration.]

On est ainsi amené à considérer quatre cas différents (classification donnée par Hickernell) précisés tableau I.

Dans ce qui suit nous analysons en détail le cas A et nous nous contentons de donner les résultats relatifs aux trois autres cas.

La prise en compte de l'épaisseur $E$ du substrat n'est utile que si elle est du même ordre de grandeur que $L$, ce qui n'est pas le cas usuel. On a calculé que les deux
Tableau I. - Classification des transducteurs à couches minces.

[Thin film transducer classification.]

\begin{tabular}{|c|c|c|}
\hline CA S & INTERFACE $x_{3}=0$ & INTERFACE $x_{3}=-H$ \\
\hline A & ELECTRODES & R I E N \\
\hline B & ELECTRODES & MEtalLI IATION \\
\hline C & R I E N & ELECTRODES \\
\hline$D$ & METALLISATION & ÉLECTRODES \\
\hline
\end{tabular}

problèmes coïncident lorsque $E$ et $L$ vérifient $\exp (-2 \pi E / L) \ll 1$.

2.2 LeS POTENTIELS. - Nous examinons successivement chacun des milieux. Le vide et le substrat sont caractérisés par leurs constantes diélectriques, respectivement $\varepsilon_{0}$ et $\varepsilon_{\mathrm{s}}$. La couche anisotrope a un tenseur diélectrique diagonal (compte tenu des axes choisis) :

$$
\varepsilon_{11}=\varepsilon_{22} ; \quad \varepsilon_{33} \neq \varepsilon_{11} \text {. }
$$

Nous nous plaçons dans le cas de couches à faible piézoélectricité ( $\mathrm{ZnO}$ ou $\mathrm{CdS}$ par exemple) de telle sorte que, lors du fonctionnement du transducteur, le champ électrique au voisinage des électrodes est essentiellement le champ appliqué. Dans ces conditions, il est correct de négliger les constantes de piézoélectricité ; le problème devient ainsi purement électrostatique.

2.2.1 Potentiel dans le substrat. - La fonction $\varphi_{\mathrm{s}}\left(x_{1}, x_{3}, t\right)$ doit vérifier

$$
\frac{\partial^{2} \varphi_{\mathrm{s}}}{\partial x_{1}^{2}}+\frac{\partial^{2} \varphi_{\mathrm{s}}}{\partial x_{3}^{2}}=0
$$

La périodicité et l'imparité de $\varphi_{\mathrm{s}}$ se traduisent par :

$$
\begin{aligned}
\varphi_{\mathrm{s}}\left(x_{1}+\frac{L}{2}\right) & =-\varphi_{\mathrm{s}}\left(x_{1}\right) \quad\left(\forall x_{3}\right) \\
\varphi_{\mathrm{s}}\left(x_{1}=0\right) & =0
\end{aligned}
$$

Par suite :

$\varphi_{\mathrm{s}}\left(x_{1}, x_{3}, t\right)=\sum_{n=1}^{\infty} \Phi_{\mathrm{s} n} \exp \left(-k_{n} x_{3}\right) \sin \left(k_{n} x_{1}\right) \exp (j \omega t)$

en posant $k_{n}=\frac{2 \pi}{L}(2 n-1)$ et $\Phi_{\text {sn }}$ désignant le $(2 n-1)$-ième coefficient de Fourier de $\varphi_{s}\left(x_{1}, 0,0\right)$. 
2.2.2 Potentiel dans le vide. - De la même façon $\varphi_{\mathrm{v}}\left(x_{1}, x_{3}, t\right)$ doit vérifier l'équation de Laplace dans le - vide. Par suite

$$
\begin{aligned}
\varphi_{\mathrm{v}}\left(x_{1}, x_{3}, t\right)=\sum_{n=1}^{\infty} \Phi_{\mathrm{v} n} \exp \left(k_{n}\left(x_{3}+H\right)\right) \times \\
\times \sin \left(k_{n} x_{1}\right) \exp (j \omega t)
\end{aligned}
$$

où les $\Phi_{\mathrm{v} n}$ désignent les coefficients de Fourier de la fonction $\varphi_{\mathrm{v}}\left(x_{1},-H, 0\right)$.

2.2.3 Potentiel dans la couche. - L'équation $\operatorname{div} \mathbf{D}=0$ admet les solutions élémentaires

$$
\exp \left( \pm r k x_{3}\right) \sin \left(k x_{1}\right) \exp (j \omega t)
$$

en posant $r=\left(\varepsilon_{11} / \varepsilon_{33}\right)^{1 / 2}$.

Par conséquent, on peut écrire

$$
\begin{aligned}
\varphi_{\mathrm{c}}\left(x_{1}, x_{3}, t\right) & =\sum_{n=1}^{\infty}\left(\Phi_{\mathrm{c} 1 n} \exp \left(r k_{n} x_{3}\right)+\Phi_{\mathrm{c} 2 n} \times\right. \\
& \left.\times \exp \left(-r k_{n} x_{3}\right)\right) \sin \left(k_{n} x_{1}\right) \exp (j \omega t) .
\end{aligned}
$$

3. Interfaces. - 3.1 CAS A. - Les différentes expressions du potentiel électrique vérifient certaines relations aux interfaces. Pour $x_{3}=0$, la continuité du potentiel (ou de la composante $E_{1}$ du champ électrique) s'écrit :

$$
\Phi_{\mathrm{s} n}=\Phi_{\mathrm{c} 1 n}+\Phi_{\mathrm{c} 2 n} .
$$

Par ailleurs la discontinuité de $D_{3}$ composante normale de l'induction électrique $D$, s'écrit :

$$
D_{\mathrm{s} 3}\left(x_{1}, 0_{+}, t\right)-D_{\mathrm{c} 3}\left(x_{1}, 0_{-}, t\right)=\sigma\left(x_{1}, t\right)
$$

$\sigma\left(x_{1}, t\right)$ étant la densité de charge à l'interface $x_{3}=0$, par unité de longueur selon $\mathrm{Ox}_{2}$. La propriété essentielle de cette fonction est d'être nulle entre les électrodes. Mais l'hypothèse d'électrodes idéales entraîne l'apparition de champs infinis aux bords des électrodes, c'est-à-dire en

$$
x_{1}=\frac{L}{4}(1 \pm a)+p \frac{L}{2} \text { pour } p \text { entier } .
$$

La fonction $\sigma\left(x_{1}\right)$ tend vers l'infini en ces points et n'admet pas un développement de Fourier convergent.

Par contre la fonction charge, $q\left(x_{1}, t\right)$, primitive de $\sigma\left(x_{1}\right)$, admettant une valeur moyenne nulle, peut s'écrire :

$$
q\left(x_{1}, t\right)=\exp (j \omega t) \sum_{n=1}^{\infty} q_{n} \cos \left(k_{n} x_{1}\right) .
$$

Dans ces conditions la discontinuité de $D_{3}$ en $x_{3}=0$ se traduit par

$$
\varepsilon_{\mathrm{c}}\left(\Phi_{\mathrm{c} 1 n}-\Phi_{\mathrm{c} 2 n}\right)+\varepsilon_{\mathrm{s}} \Phi_{\mathrm{s} n}=-q_{n}
$$

avec

$$
\varepsilon_{\mathrm{c}}=\left(\varepsilon_{11} \varepsilon_{33}\right)^{1 / 2} .
$$

Pour $x_{3}=-H$, la continuité du potentiel s'exprime par

$$
\Phi_{\mathrm{v} n}=\Phi_{\mathrm{c} 1 n} \exp \left(-r k_{n} H\right)+\Phi_{\mathrm{c} 2 n} \exp \left(r k_{n} H\right)
$$

et celle de $D_{3}$ par

$$
\varepsilon_{0} \Phi_{\mathrm{v} n}=\varepsilon_{\mathrm{c}}\left[\Phi_{\mathrm{c} 1 n} \exp \left(-r k_{n} H\right)-\Phi_{\mathrm{c} 2 n} \exp \left(r k_{n} H\right)\right] .
$$

Les équations (4), (5), (6) et (7) se condensent matriciellement en :

$$
\left[\begin{array}{cccc}
1 & -1 & -1 & 0 \\
\varepsilon_{\mathrm{s}} & \varepsilon_{\mathrm{c}} & -\varepsilon_{\mathrm{c}} & 0 \\
0 & \exp \left(-r k_{n} H\right) & \exp \left(r k_{n} H\right) & -1 \\
0 & \varepsilon_{\mathrm{c}} \exp \left(-r k_{n} H\right) & -\varepsilon_{\mathrm{c}} \exp \left(r k_{n} H\right) & -\varepsilon_{0}
\end{array}\right] \cdot\left[\begin{array}{l}
\Phi_{\mathrm{s} n} \\
\Phi_{\mathrm{c} 1 n} \\
\Phi_{\mathrm{c} 2 n} \\
\Phi_{\mathrm{v} n}
\end{array}\right]=\left[\begin{array}{c}
0 \\
-q_{n} \\
0 \\
0
\end{array}\right]
$$

On constate que les coefficients $\Phi_{\mathrm{s} n}, \Phi_{\mathrm{c} 1 n}, \Phi_{\mathrm{c} 2 n}$ et $\Phi_{\mathrm{v} n}$ sont proportionnels à $-q_{n}$.

En particulier

$$
\Phi_{\mathrm{s} n}=\frac{-q_{n}}{\varepsilon_{n}^{\mathbf{A}}}
$$

en posant

$$
\varepsilon_{n}^{\mathrm{A}}=\frac{\varepsilon_{\mathrm{c}}\left(\varepsilon_{\mathrm{s}}+\varepsilon_{0}\right)+\left(\varepsilon_{\mathrm{c}}^{2}+\varepsilon_{\mathrm{s}} \varepsilon_{0}\right) \text { th }\left(r k_{n} H\right)}{\varepsilon_{\mathrm{c}}+\varepsilon_{0} \operatorname{th}\left(r k_{n} H\right)} .
$$

Les coefficients $\varepsilon_{n}^{A}$ ont la dimension d'une constante diélectrique. Comme ils établissent un lien entre les fonctions $q\left(x_{1}\right)$ et $\varphi\left(x_{1}\right)$ à l'interface contenant les électrodes $\left(x_{3}=0\right)$, le système considéré est alors traité de la même façon qu'un système à une seule interface. Il en serait de même pour un système multicouches tels que ceux décrits en référence [6].

3.2 CAs B. - (Electrodes en $x_{3}=0$; métallisation en $x_{3}=-H$.) Les conditions aux interfaces sont simplifiées. Les relations (4) et (5), pour $x_{3}=0$, demeurent. 
La nullité de $\varphi_{\mathrm{c}}$ et $\varphi_{\mathrm{v}}$ en $x_{3}=-H$, se traduit par

$$
\frac{\Phi_{\mathrm{c} 2 n}}{\Phi_{\mathrm{c} 1 n}}=-\exp \left(-2 r k_{n} H\right) .
$$

On obtient finalement

avec

$$
\Phi_{\mathrm{s} n}=\frac{-q_{n}}{\varepsilon_{n}^{\mathrm{B}}}
$$

$$
\varepsilon_{n}^{\mathbf{B}}=\varepsilon_{\mathrm{s}}+\frac{\varepsilon_{\mathrm{c}}}{\operatorname{th}\left(r k_{n} H\right)} .
$$

3.3 CAS C. - (Electrodes en $x_{3}=-H$.) Ce cas, analogue au cas A, conduit au système

$$
\left[\begin{array}{cccc}
-1 & -1 & -1 & 0 \\
\varepsilon_{\mathrm{s}} & \varepsilon_{\mathrm{c}} & -\varepsilon_{\mathrm{c}} & 0 \\
0 & \exp \left(-r k_{n} H\right) & \exp \left(r k_{n} H\right) & -1 \\
0 & -\varepsilon_{\mathrm{c}} \exp \left(-r k_{n} H\right) & \varepsilon_{\mathrm{c}} \exp \left(r k_{n} H\right) & \varepsilon_{0}
\end{array}\right]\left[\begin{array}{l}
\Phi_{\mathrm{s} n} \\
\Phi_{\mathrm{c} 1 n} \\
\Phi_{\mathrm{c} 2 n} \\
\Phi_{\mathrm{v} n}
\end{array}\right]=\left[\begin{array}{r}
0 \\
0 \\
0 \\
-q_{n}
\end{array}\right]
$$

et par conséquent

$$
\Phi_{\mathrm{v} n}=\frac{-q_{n}}{\varepsilon_{n}^{\mathrm{c}}}
$$

avec

$$
\varepsilon_{n}^{\mathrm{c}}=\frac{\varepsilon_{\mathrm{c}}\left(\varepsilon_{0}+\varepsilon_{\mathrm{s}}\right)+\left(\varepsilon_{\mathrm{c}}^{2}+\varepsilon_{0} \varepsilon_{\mathrm{s}}\right) \text { th }\left(r k_{n} H\right)}{\varepsilon_{\mathrm{c}}+\varepsilon_{\mathrm{s}} \text { th }\left(r k_{n} H\right)} .
$$

3.4 CAS D. - (Electrodes en $x_{3}=-H$, métallisation en $x_{3}=0$.) La nullité de $\varphi_{\mathrm{c}}$ et $\varphi_{\mathrm{s}}$ en $x_{3}=0$, donne

$$
\Phi_{\mathrm{c} 1 n}=-\Phi_{\mathrm{c} 2 n}
$$

quant aux équations relatives à l'interface $x_{3}=-H$, ce sont celles du $\S 3.3$. Il en résulte :

$$
\Phi_{\mathrm{v} n}=\frac{-q_{n}}{\varepsilon_{n}^{\mathrm{D}}} \text { avec } \varepsilon_{n}^{\mathrm{D}}=\varepsilon_{0}+\frac{\varepsilon_{\mathrm{c}}}{\operatorname{th}\left(r k_{n} H\right)} .
$$

Remarquons la convergence rapide de $\varepsilon_{n}$ vers une valeur positive, lorsque $n$ augmente, et ce dans tous les cas.

4. Résolution. - Quel que soit le cas envisagé nous avons la relation

$$
\Phi_{n}=-\frac{q_{n}}{\varepsilon_{n}}
$$

entre les coefficients de Fourier de la charge $q\left(x_{1}\right)$ et du potentiel $\varphi\left(x_{1}\right)$ à l'interface contenant les électrodes. Lorsque le coefficient de métallisation est donné, on peut résoudre le problème, c'est-à-dire déterminer $q$ et $\varphi$. On obtient également la capacité $C$ d'une paire d'électrodes par unité de longueur.

Nous utilisons les conditions aux limites, à l'interface des électrodes, sur un quart de période seulement, du fait des symétries (Fig. 2).

On subdivise les zones métallisée $\frac{L}{4}(1-a)<x_{1}<\frac{L}{4}$ et non métallisée $0<x_{1}<\frac{L}{4}(1-a)$ respectivement en $M$ et $N$ segments égaux; ainsi :

$$
\frac{a}{M}=\frac{1-a}{N}=\frac{1}{M+N}
$$

Dans le cas où $a$ n'est pas une fraction rationnelle conduisant à $M+\mathrm{N}$ de l'ordre de 30 (cf. $\S 5$ ), on effectue les calculs qui suivent pour deux valeurs encadrant $a$ et on procède par interpolation. 


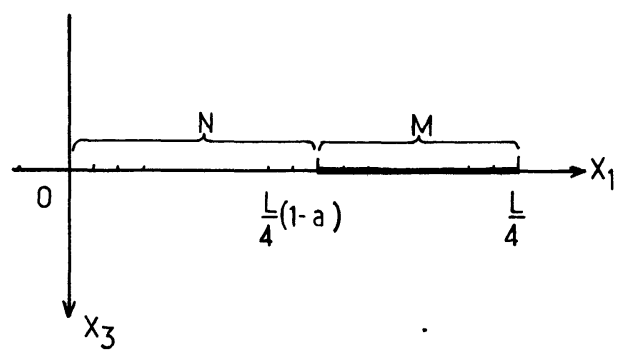

Fig. 2. - Conditions aux limites.

[Boundary conditions.]

Pour donner un même poids à tous les points de l'intervalle $\left[0, \frac{L}{4}\right]$, les conditions aux limites sont écrites au milieu de chaque segment, c'est-à-dire : pour la partie non métallisée, aux abscisses

$$
(p-0,5) \frac{L}{4(M+N)} \text { avec } 1 \leqslant p \leqslant N
$$

et sur l'électrode, aux abscisses

$$
\frac{L}{4}(1-a)+(p-0,5) \frac{L}{4(M+N)} \text { avec } 1 \leqslant p \leqslant M .
$$

Dans l'intervalle $\left[0, \frac{L}{4}(1-a)\right]$, non métallisé, on écrit que la fonction $q\left(x_{1}\right)$ est constante et vaut $-Q / 2$ où $Q$ désigne la charge d'une électrode, par unité de longueur selon $o x_{2}$.

$$
q\left(\frac{(p-0,5) L}{4(M+N)}\right)=-\frac{Q}{2} \text { pour } 1 \leqslant p \leqslant N
$$

Sur l'électrode, c'est le potentiel qui est constant

$$
\Phi\left(\frac{L}{4}(1-a)+\frac{(p-0,5) L}{4(M+N)}\right)=\frac{V}{2} \text { pour } 1 \leqslant p \leqslant M .
$$

Compte tenu de l'inconnue $Q$ ces $M+N$ équations permettent de calculer $M+N-1$ coefficients $q_{n}$. Ainsi, les développements en série de Fourier sont-ils considérés jusqu'à l'ordre $2(M+N)-3$. On obtient finalement le système linéaire suivant :

$$
\left[\begin{array}{c:c}
T_{m n}=\frac{1}{\varepsilon_{n}} \sin \left(\frac{\pi}{2}(2 n-1)\left(1-a+\frac{m-0,5}{M+N}\right)\right) & 0 \\
\hdashline & 0 \\
T_{m n}=\cos \left(\frac{\pi}{2}(2 n-1)\left(\frac{m-M-0,5}{M+N}\right)\right) & 1 \\
\hdashline & 1
\end{array}\right]=-\frac{V}{2}\left[\begin{array}{l}
q_{1} \\
q_{M} \\
q_{M+1} \\
q_{M+N-1} \\
Q / 2
\end{array}\right]\left[\begin{array}{l}
Y_{m}=1 \\
\hdashline
\end{array}\right]
$$

On résout, en fait, le système linéaire

$$
T_{m n} \cdot X_{n}=Y_{m}
$$$$
q_{n}=-\frac{V}{2} X_{n} \text { pour } 1 \leqslant n \leqslant M+N-1
$$

dans lequel les équations sont toutes multipliées par

$-2 / V$. On a donc

et $Q=-V X_{M+N}$.

REVUE DE PHYSIQUE APPLIQUÉE. - T. 17, No 2, FÉVRIER 1982 
La capacité par période $L$ et par unité de longueur, notée $C$, vaut alors

$$
\frac{Q}{V}=-X_{M+N}
$$

La résolution du système linéaire donne donc directement cette caractéristique sans avoir recours à une série numérique [5-6] ou à une formule algébrique [4-7].

5. Résultats. - Nous avons résolu numériquement le système linéaire à l'aide d'un programme FORTRAN qui effectue également le calcul de la répartition des charges et du potentiel en tout point de la structure. La simplicité de la méthode autorise l'utilisation d'un ordinateur de petite taille (UNIDATA 7720), mais la double précision (8 octets par valeur numérique) est nécessaire pour approcher les valeurs de capacité au millième, ce qui semble suffisant, compte tenu de l'incertitude sur les constantes diélectriques. Dans ces conditions, la mémoire nécessaire ne dépasse pas 10 Koctets et le calcul d'une valeur de la capacité ne dure que quelques secondes.

Pour un cas donné (configuration, $a$ et $K H=2 \pi H / L$ choisis) la précision de l'évaluation de la capacité dépend de la dimension $M+N$ du système linéaire. Pour des valeurs trop faibles $(<5)$ les développements en série de Fourier n'ont pas assez de termes; pour des valeurs élevées $(>40)$ notre programme ne résout plus le système linéaire de façon satisfaisante; il faudrait alors passer en multiple précision.

En observant l'évolution des résultats lorsque $M+N$ augmente, nous avons déterminé l'incertitude relative sur $C$; nous la donnons, figure 3 , pour les valeurs 10,20 et 30 de $M+N, a$ variant de 0,1 à 0,9

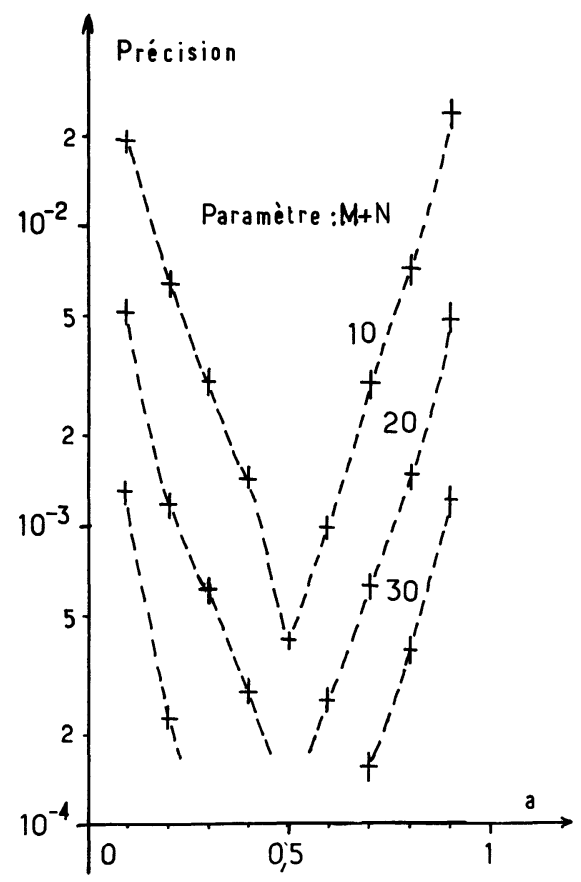

Fig. 3. - Précision de la méthode.

[Method accuracy.]

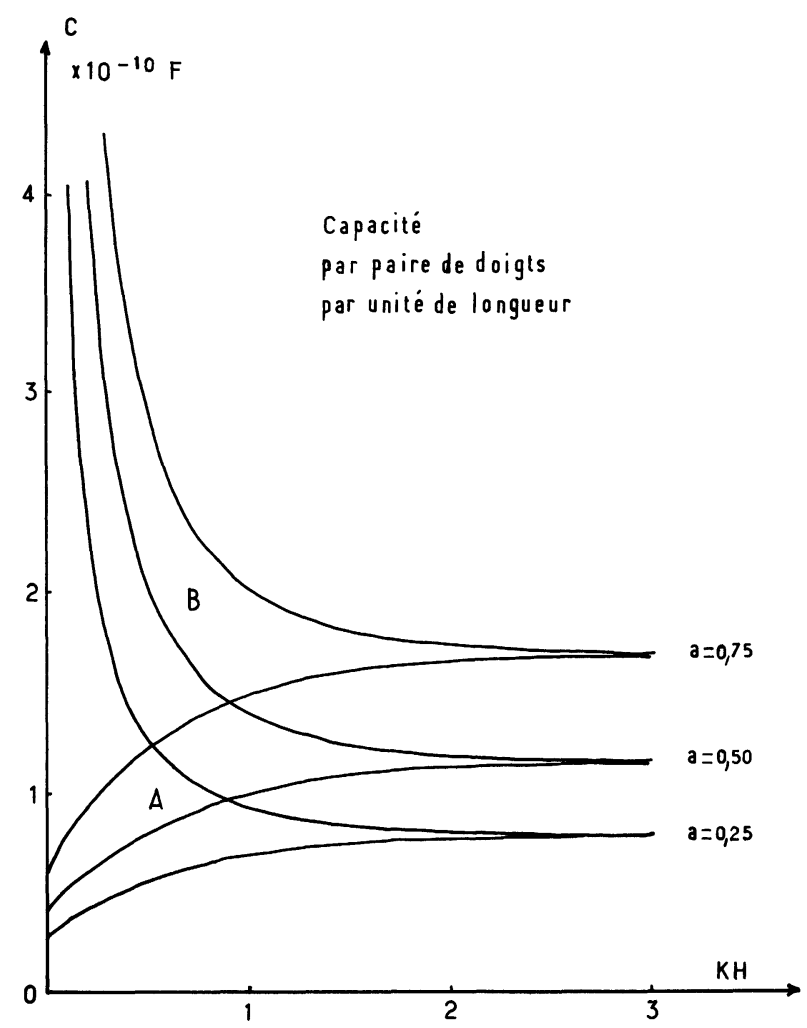

Fig. 4. - Variations de la capacité en fonction de $K H$ (paramètre : $a$ ).

[Capacitance as a function of $K H(a$ parameter $)$.]

avec un pas de 0,1. Pour les valeurs intermédiaires de $a$ la précision est éventuellement moins bonne du fait de l'interpolation.

Il apparait que, pour les valeurs usuelles du coefficient de métallisation $(0,2<a<0,8)$, l'erreur relative sur la capacité est inférieure au millième pour $M+N=30$. Tous les résultats présentés ont été obtenus dans ces conditions.

Le cas présenté ici est celui de couches de sulfure de cadmium CdS déposées sur un substrat en silice. Les données numériques sont les suivantes :

$$
\begin{array}{lll}
\text { pour le vide } & \varepsilon_{0}=8,842 \times 10^{-12} \mathrm{~F} / \mathrm{m} \\
\text { pour la silice } & \varepsilon_{\mathrm{s}}=3,298 \times 10^{-11} \mathrm{~F} / \mathrm{m} \\
\text { pour le Cds } & \varepsilon_{11}=7,98 \times 10^{-11} \mathrm{~F} / \mathrm{m} \\
\text { (Réf. [9]) } & \varepsilon_{33}=8,43 \times 10^{-11} \mathrm{~F} / \mathrm{m} .
\end{array}
$$

Les variations de la capacité, en fonction de $K H$, dans les cas A et $\mathrm{B}$, sont données figure 4 pour trois coefficients de métallisation. Il est clair que pour $K H>3$, la couche se comporte comme un milieu d'épaisseur infinie (faible variation de la capacité; courbes des cas A et B confondues). L'influence du coefficient $a$ apparaît, figure 5 , dans le cas $\mathrm{A}$, pour 5 valeurs différentes de $K H$.

Nous présentons enfin le tracé des fonctions introduites $\varphi\left(x_{1}\right)$ et $q\left(x_{1}\right)$ à l'interface $x_{3}=0$, dans le cas $\mathrm{A}$ $(a=0,5 ; K H=1)$; ces fonctions sont définies à $V / 2$ près. La figure 6 est complétée par la densité de 


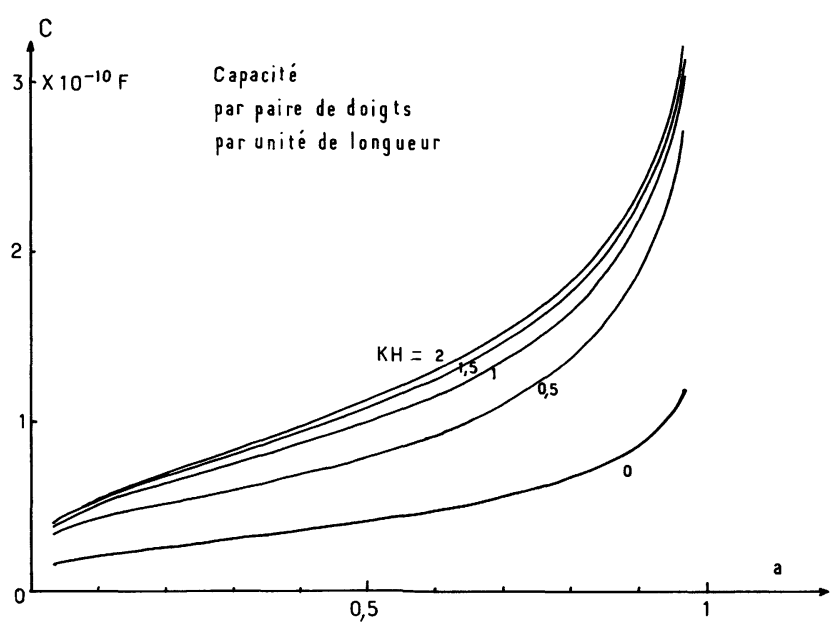

Fig. 5. - Variation de la capacité en fonction du coefficient de métallisation $a$ (paramètre $K H$ ).

[Capacitance as a function of metallization factor $a(\mathrm{KH}$ parameter).]

charge $\sigma\left(x_{1}\right)$ au facteur $V / L$ près. En fait nous portons, sur la zone métallisée, la somme :

$$
-\sum_{n=1}^{M+N-1} q_{n} k_{n} \sin \left(k_{n} x_{1}\right)
$$

dont la convergence est, on le sait, problématique.

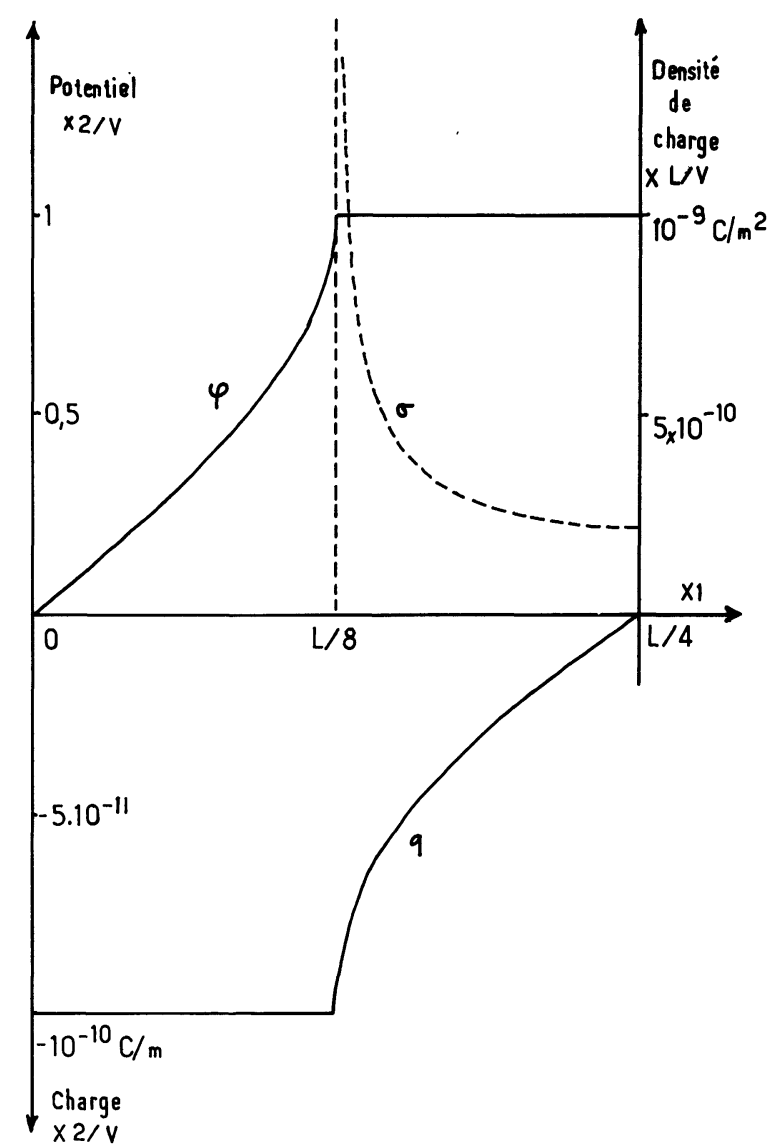

Fig. 6. - Répartition du potentiel $\varphi$, de la charge $q$ et de la densité de charge $\sigma$ à l'interface $x_{3}=0$.

[Distribution of potential $\varphi$, charge $q$ and charge density $\sigma$ at $x_{3}=0$ interface.]
Nous avons comparé ces résultats à ceux déjà publiés [5]. L'approximation réalisée par leurs auteurs (densité de charge uniforme sur les doigts) n'autorise pas une bonne précision. Des écarts de 10 à $20 \%$ sont enregistrés sur les valeurs de capacités pour $a>0,5$ alors que l'écart entre nos mesures et nos calculs n'excède jamais $5 \%$.

Les calculs de Engan représentent le cas limite $K H=0$ des configurations A et $\mathrm{C}$. La formule de la capacité donnée par l'auteur [4] est vérifiée par nos calculs.

Par ailleurs, Farnell a utilisé la méthode des éléments finis pour calculer la capacité et la répartition des potentiels dans des structures isotropes. Notre programme, appliqué aux données correspondantes, fournit des variations de capacité identiques à celles présentées en référence [8].

6. Génération d'ondes. - La création d'ondes se propageant dans les deux directions de l'axe $o x_{1}$, de part et d'autre d'un transducteur limité dans ces directions, est envisageable si la piézoélectricité de la couche n'est plus négligée. On admettra que pour un transducteur comportant un nombre suffisant de doigts (10 à 30) nos résultats sont encore valables.

Les ondes créées peuvent être du type de surface (ou de Rayleigh généralisées) ou de volume (dans ce cas, le substrat d'épaisseur finie sert de guide à cette propagation) [10].

Pour les deux composantes du champ électrique responsables des déplacements, au niveau des transducteurs, les coefficients de Fourier sont, dans le cas B :

$E_{1 n}\left(x_{1}, x_{3}\right)=\frac{k_{n} q_{n}}{\varepsilon_{n}} \cdot \frac{\operatorname{sh}\left[r k_{n}\left(x_{3}+H\right)\right]}{\operatorname{sh}\left(r k_{n} H\right)} \cos \left(k_{n} x_{1}\right)$

$E_{3 n}\left(x_{1}, x_{3}\right)=\frac{k_{n} q_{n}}{\varepsilon_{n}} \cdot \frac{\operatorname{ch}\left[r k_{n}\left(x_{3}+H\right)\right]}{\operatorname{sh}\left(r k_{n} H\right)} \sin \left(k_{n} x_{1}\right)$.

L'existence d'harmoniques spatiaux, selon 'ox entraîne la possibilité de propagation avec une longueur d'onde de phase $\frac{L}{2 n-1}(n \geqslant 1)$.

L'examen des relations (12) montre que pour $K H$ fixé et en un point donné $\left(x_{1}, x_{3}\right)$ de la couche, le coefficient $k_{n} q_{n} / \varepsilon_{n}$ mesure l'amplitude relative de l'harmonique $2 n-1$ lorsque $a$ varie.

La variation de $k_{n} q_{n} / \varepsilon_{n}$ en fonction de $a$ (cas B; $K H=0,5)$ est représentée figure 7 .

On voit clairement que certains harmoniques peuvent ne pas exister (par exemple l'harmonique 5 pour $a=0,32$ et $a=0,71$ ), alors que l'harmonique 1 , lui, existe toujours.

Enfin, on vérifie la décroissance des $E_{1 n}$ et $E_{3 n}$ lorsque $x_{3}$ varie de 0 à $H$ : les harmoniques sont essentiellement engendrés au voisinage des doigts et leur importance décroît, quand leur ordre augmente [8].

Ces résultats sont à rapprocher de celui donné par Engan [4] dans son étude de la génération des ondes de surface sur un piézoélectrique. Nous retrouvons 


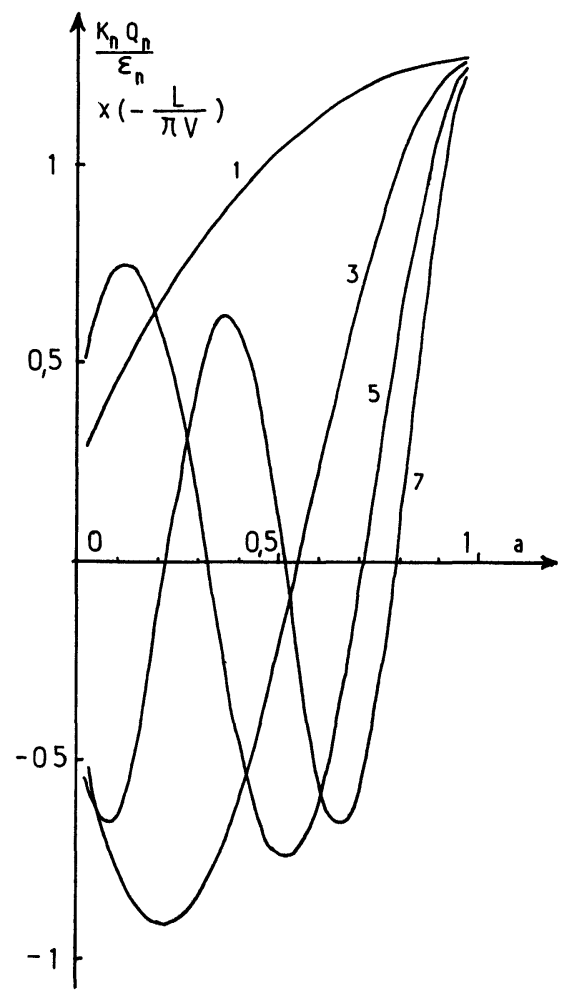

Fig. 7. - Amplitude des harmoniques spatiaux en fonction de $a$.

[Amplitude of spatial harmonics as a function of $a$.]

exactement sa solution en prenant $K H$ nul ou infini dans les deux cas $\mathrm{A}$ et $\mathrm{C}$ (la structure ne possède plus qu'une seule interface). Par contre l'influence de $K H$ se traduit par le déplacement des zéros des fonctions $\frac{k_{n} q_{n}}{\varepsilon_{n}}(a)$; par exemple l'harmonique 3 s'annule, dans le cas proposé (Fig. 7), pour $a=0,55$ au lieu de 0,50.

7. Conclusion. - Présentée pour l'étude des structures en monocouche, notre méthode est générale. Elle peut s'appliquer aux traditionnels cristaux piézoélectriques ainsi qu'aux structures multicouches; dans chaque cas, il suffit de recalculer les coefficients $\varepsilon_{n}$ adaptés. Pour étudier un système multi-électrodes (par exemple, à électrodes doubles, [11]) seuls les termes de la matrice du système linéaire sont à modifier; le programme informatique conserve sa structure initiale.

Par ailleurs, l'algorithme présente les avantages suivants :

- il n'utilise que les résultats classiques de l'analyse de Fourier,

- l'introduction de la fonction charge $q\left(x_{1}\right)$, à l'interface comprenant les électrodes, à la place de la densité de charge $\sigma\left(x_{1}\right)$, conduit à des séries plus rapidement convergentes, donc à des calculs numériques plus précis et moins volumineux,

- la précision des résultats ne dépend que des moyens informatiques mis en ouvre puisque les calculs analytiques ne comportent aucune approximation.

Ainsi, sur un ordinateur de petite taille et pour une précision du millième, notre programme ne requiert que quelques secondes de temps d'exécution et qu'une dizaine de Koctets de mémoire; il est donc tout à fait possible de l'implémenter sur un micro-ordinateur.

\section{Bibliographie}

[1] Dieulesaint, E., Royer, D., Ondes élastiques dans les solides (Masson Ed., Paris) 1974.

[2] Hickernell, F. S., Piezoelectric film surface wave transducers in : Acoustic surface wave and acoustooptic devices (Optosonic press, New York) 1971, p. 31-41.

[3] Hickernell, F. S., Triode sputtered zinc oxide surface elastic wave transducers, J. Appl. Phys. 44 (1973) 1061-1071.

[4] ENGaN, H., Excitation of elastic surface wave by spatial harmonics of interdigital transducers, I.E.E.E. Trans. Electron. Devices ED 16 (1969) 1014-1017.

[5] Kino, G. S., Wagers, R. S., Theory of interdigital couplers on non piezoelectric substrates, J. Appl. Phys. 44 (1973) 1480-1488.

[6] Venema, A., Dekkers, J. J. M., Humphryes, R. F., Static capacitance calculations for a surface acoustic wave interdigital transducer in multilayered media, I.E.E.E. Trans. Microwaves Th. Tech. MTT 26 (1978) 294-297.

[7] Ponamgi, S. R., Tuan, H. S., Theoretical calculation of capacitance of an I.D.T. over a piezoelectric layered structure, I.E.E.E. Trans. Sonics Ultrasonics, SU 21 (1974) 125-127.

[8] Farnell, G. W., Cermak, I. A., Silvester, P., Wong, S. K., Capacitance and field distribution for interdigital surface wave transducers, I.E.E.E. Trans. Sonics Ultrasonics SU 17 (1970) 188-195.

[9] JAFFe, H., Berlincourt, D., Piezoelectric transducers materials, Proc. I.E.E.E. 53 (1965) 1372-1386.

[10] Nalamwar, A. L., Wagers, R. S., Epstein, M., Efficient bulk-wave excitation by interdigital transducers in layered media, J. Appl. Phys. 48 (1977) 2175-2178.

[11] ENGAN, H., Surface acoustic wave multielectrode transducers, I.E.E.E. Trans. Sonics Ultrasonics SU 22 (1975) 395-401. 